\title{
Habituation and recovery of vascular responses in calf and forearm and of the level of pain sensation during the cold pressor test in man
}

\author{
A. Zbrożyna and D. Westwood
}

Department of Physiology, The Medical School, University of Birmingham, Birmingham B15 2TJ, England

Eur J Appl Physiol (1990) 61:106-111

On page 110, left column, the sentence beginning three lines from the bottom should read: "These changes were observed before attack or an attempt to escape". 